\title{
Role of Multidetector CT in Blunt Abdominal Trauma
}

\author{
Praveen Kumar John ${ }^{1}$, Arif Khans ${ }^{2}$, Ganesh K ${ }^{3}$, Francis N. P. Monteiro ${ }^{4}$ \\ ${ }^{1}$ Associate Professor, Department of Radio-Diagnosis, A.J. Institute of Medical Sciences and Research Centre, NH 17, Kuntikana, Mangalore -575004. ${ }^{2}$ Assistant \\ Professor, Department of Radio-Diagnosis, Sreemookambika Medical College, Thiruvananthapuram, ${ }^{3}$ Professor, Department of Radio-Diagnosis, A.J. Institute of \\ Medical Sciences and Research Centre, NH 17, Kuntikana, Mangalore -575004, ${ }^{4}$ Professor, Department of Forensic Medicine \& Toxicology, A.J. Institute of Medical \\ Sciences and Research Centre, NH 17, Kuntikana, Mangalore -575004.
}

\section{Abstract}

Background: Computed Tomographic (CT) evaluation of Abdomen has become the mainstay of management of all types of trauma to abdomen, in particularly blunt trauma abdomen. This change is mainly attributable to the ability of CT scanning to produce most detailed images of the areas of study or interest. CT can determine the source of hemorrhage. However CT is not that accurate in detecting all types of injuries for example, mesenteric injuries and small bowel injuries are sometimes detected surgically only and missed in CT imaging. Aims and Objectives: 1.To study with the aid of multi detector CT (MDCT), different traumatic pathologies of abdomen in blunt trauma. 2. To corelate imaging findings with surgical findings or clinical data. 3. To evaluate the accuracy of CT in detecting various lesions by statistical analysis. Subjects and Methods: The study was conducted in the Department of Radiodiagnosis at A.J Institute of Medical Sciences and Research Center, Mangalore. Patients with history of Road Traffic Accidents, Fall, or assault or other causes where clinically blunt trauma of abdomen is suspected and referred for CT abdomen were studied using Multidetector CT (MDCT) A total of 56 patients with abdominal trauma who underwent Computed Tomography (CT) examination were included. CT findings were compared with surgical findings in 37 operated cases. Results: A total number of 56 cases were selected based on Ultrasound and Clinical inferences for CT evaluation. 37 cases underwent surgery and remaining were managed conservatively. On correlating with surgical findings MDCT has a sensitivity of $80 \%$, specificity of $95 \%$ for detecting solid organ injury and the PPV and NPV were $97 \%$ and 70\%respectively. Meanwhile for detection of Hollow Viscus Injury (HVI) sensitivity and specificity were $75 \%$ and $96 \%$ respectively. Conclusion: MDCT is the gold standard for evaluation of blunt trauma abdomen. Prompt imaging in proper technique and expertise in reporting improves the detection rate and accuracy of all types of abdominal injuries associated with blunt trauma abdomen. While MDCT diagnosis of solid organ injury is highly accurate, the detection rate of hollow viscus injury and bladder injury is not optimal. Bladder injuries particularly extra-peritoneal type should be further analyzed by ascending urethrogram to confirm it as well as to rule out urethral injury.

Keywords: Computed tomography, Blunt trauma, Abdomen, Injuries

Corresponding Author: Dr Arif Khan S, Alif Villa,614/15, Edamanakuzhi,Thekkekulam road, Balaramapuram, Trivandum 695501.

Received: April 2018

Accepted: June 2018

\section{Introduction}

The economic impact, morbidity and mortality resulting from trauma in general, and blunt abdominal trauma in particular, are substantial. Blunt abdominal trauma makes up $75 \%$ of all blunt trauma and is the most common example of this injury. ${ }^{[1]}$ Unlike penetrating abdominal trauma, where management is largely determined clinically, the diagnosis of blunt abdominal injury by clinical examination is unreliable, particularly in patients with a decreased level of consciousness. ${ }^{[2]}$ Confirmation of the presence or absence of injury therefore relies largely on the use of diagnostic adjuncts.

In a large prospective observational study of patients with blunt poly-trauma but no clinical signs of injury found that radiological evidence of abdominal injury was present in almost $10 \%$ of patients. ${ }^{[5]}$ Recent consensus guidelines suggest that even in a low threshold of clinical suspicion of blunt trauma abdomen CT evaluation should be ordered without hesitation once we weigh the risks versus benefits of the study. ${ }^{[4-5]}$

Accurate imaging facilitates selection for non-operative management, where appropriate, and reduces nontherapeutic laparotomy rates. ${ }^{[3]}$ The main first line investigations are ultrasound, diagnostic peritoneal lavage, and computed tomography.. CT scanning often provides the most detailed images of traumatic pathology and may assist in determination of operative intervention7.Unlike Diagnostic peritoneal lavage (DPL) or focused abdominal sonography for trauma (FAST), CT can determine the source of hemorrhage. ${ }^{[5]}$ Mesenteric injuries and small bowel injuries are sometimes detected surgically only and missed by CT imaging reports. ${ }^{[7]}$ The purpose of this study is to assess the sensitivity as well as specificity of MDCT scan abdomen in detecting injuries by correlating with surgical findings of the same. Ultrasound is the investigation of choice in haemodynamicallyunstable patients. Computed tomography is the investigation of choice in haemodynamically stable patients .Solid organ 
injury in haemodynamically stable patients can often be managed without surgery. ${ }^{[8]}$

\section{Objectives of the study:}

1. To study with the aid of Multi-Detector CT, different traumatic pathologies of abdomen in blunt trauma.

2. To evaluate the accuracy of CT in detecting various traumatic pathologies by statistical analysis.

3. To correlate study findings with existing research literature.

\section{Subjects and Methods}

The study was conducted in the Department of Radiodiagnosis at AJ Institute of Medical Sciences and Research Center, Mangalore, Karnataka, Mangalore. Patients with history of Road Traffic Accidents, fall, or assault or other causes where clinically blunt trauma of abdomen is suspected and referred for CT abdomen.

All cases underwent FAST scan in the department prior to CTscan.

\section{Inclusion Criteria}

1. Clinical suspicion of abdominal trauma.

2. All poly- trauma cases.

3. Hemodynamically stable patients.

\section{Exclusion criteria}

All hemodynamically unstable patients were excluded from CT evaluation unless stabilized. Due to large volume of blunt trauma suspected cases in our hospital, Cases with positive FAST ultrasound scan result or with CT findings were selected. Randomization was not required. So a total of 56 patients with abdominal trauma who underwent Computed Tomography (CT) examination were included. All cases underwent FAST ultrasound scan prior to CT. 37 cases out of 56 underwent explorative laparotomy. These cases were evaluated for correlation between radiological and surgical Findings. The remaining cases were evaluated in comparison with clinical outcome and ultrasound correlation. The study was conducted from August 2013 to October 2014

\section{$\underline{\mathrm{CT}}$}

$\overline{\text { Out }}$ of the 56 cases studied 40 were evaluated using 64 slice Multidetector CT (Lightspeed VCT; GE Medical Systems) and remaining 16 on 128 slice Dual Energy CT (Siemen's; Somatom, Definition). All cases underwent CT evaluation with I.V contrast injection.

The parameters used for acquisition of images in CT abdomen assessment is given below

\section{4 slice MDCT Acquisition Parameters: ${ }^{191}$}

Scan mode : Helical

Slice thickness: $5 \mathrm{~mm}$

- Pitch: $1.375 \mathrm{~mm}$

- Rotation time: $0.5 \mathrm{sec}$

- $\mathrm{mA}-300-380 \mathrm{~mA}$

- $\mathrm{kV}=120 \mathrm{kVp}$

All acquired images were reconstructed into $0.625 \mathrm{~mm}$ slice thickness using GE factory recon tool inbuilt in scanner.
Additional rendering were done for 3D assessment for vascular structures and bones whenever required using ADVANTAGE Workstation tool, GE.

\section{8 slice MDCT (DECT) Acquisition Parameters:}

Scan mode: Spiral

Slice thickness: $5 \mathrm{~mm}$

- Pitch: $0.6 \mathrm{~mm}$

- Rotation time: $0.5 \mathrm{sec}$

- $\mathrm{mA}=500-625 \mathrm{~mA}$ (eff.mAs 200-250)

- $\mathrm{kV}=120 \mathrm{kVp}$

All acquired images were reconstructed into $0.7 \mathrm{~mm}$ slice thickness and additional adjustments like 3D rendering MPR assessment etc. were done using SYNGO.VIA tool of Seimens.

\section{Contrast Media:}

Ultravist 370 (iopromide), a low molecular weight Ionic contrast was used for the study at a dose of $1 \mathrm{ml} / \mathrm{kg}$ body weight dose.

\section{Imaging Protocol}

All patients were examined pre and post administration of I. $V$ contrast

A topogramwasacquired prior to scan proper and planner was drawn from above diaphragm to below ischium for a total distance of 512 to $766 \mathrm{~mm}$ average according to patient's body build. Contrast was administered through a pre-programmed Pressure injector with a flow rate of 3$3.5 \mathrm{ml}$ per second and always were followed by $30 \mathrm{ml}$ saline push.

Auto imaging technique was used to accurate image acquisition with Region of Interest (ROI) marker kept in sub-diaphragmatic abdominal aorta. Image acquisition times for various phases are as follows

The post contrast study included following phases of image acquisition. After contrast administration

Arterial $-35 \mathrm{~s}$

Porto-venous $-45-60 \mathrm{~s}$

Delayed- $15 \mathrm{~min}$

Acquired images were analyzed by two Radiologists.Acquired CT raw images were RetroReconstructed using aforementioned factory software embedded in the Machine operating system. The sensitivity, specificity, positive predictive value and negative predictive value were calculated for each organ after surgical correlation.

\section{Results \& Discussion}

A total number of 56 cases were studied, of which 37 cases had undergone surgery. Remaining 19 patients were managed conservatively.

In our Study RTA was seen to be the leading cause of trauma, about $71 \%$ of total cases. Majority of cases, about $38 \%$ of the affected individuals belonged to the $21-30$ years of age group, followed by $31-40$ years $(23 \%)$. The male to female ratio of our study was $12.5: 1$, shows that significantly more males were affected than females.

Lone et al, ${ }^{[10]}$ in his study reports the male to female ratio 


\section{Tohn et al; Rale of Multidetector CJ in Blunt Cladominal Irouma}

was $4.4: 1$

All patients underwent FAST scan prior to the CT evaluation. On comparison with the CT finding FAST scan was found to have a sensitivity of $90 \%$ and specificity of $100 \%$ for detecting hemoperitoneum.

Table 1: Statistics of hemoperitoneum detected in FAST when compared with CT

\begin{tabular}{|c|c|}
\hline Hemoperitoneum In Fast Scan \\
\hline Total Positive & 45 \\
\hline Total Negative & 11 \\
\hline True Negative & 6 \\
\hline False Negative & 5 \\
\hline True Positive & 45 \\
\hline False Positive & 0 \\
\hline
\end{tabular}

Nauman Al Quamari et al, ${ }^{\lfloor 1\rfloor}$ found out that the sensitivity and specificity of FAST in detecting intra-abdominal free fluid was calculated to be $91.9 \%$ and $94.34 \%$ respectively.In another study by Simon Fleminget al. ${ }^{[12]}$ the sensitivity, specificity of FAST scan in detecting hemoperitoneum was $94.7 \%$ and $46.2 \%$ respectively on comparison with CT findings.

Out of 56 cases A total of 31 (55\%) cases Solid organ injury were detected in FAST scan.

Table no. 2 Frequency of injury of solid organ detected in FAST scan

\begin{tabular}{|c|c|c|}
\hline Injury & Number Of Cases & \% OF 31 \\
\hline Liver & 17 & $55 \%$ \\
\hline Spleen & 13 & $42 \%$ \\
\hline Kidneys & 8 & $25 \%$ \\
\hline Multi Organ Injury & 6 & $19 \%$ \\
\hline
\end{tabular}

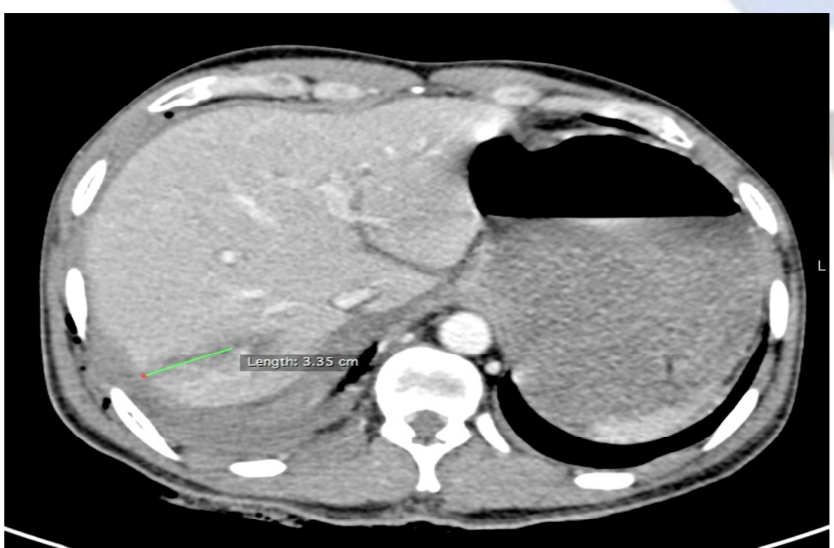

Figure 1: CECT Portal phase study, axial image showing liver laceration with sub capsular hematoma (Grade III)

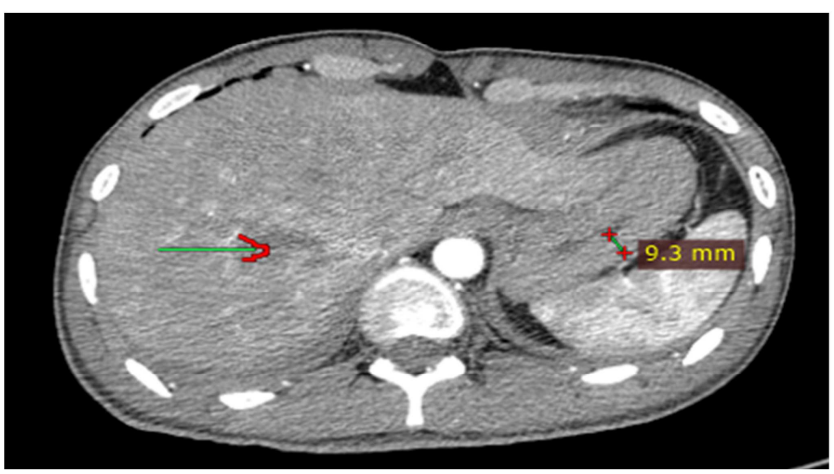

Figure 2: CECT Arterial phase study, axial images showing

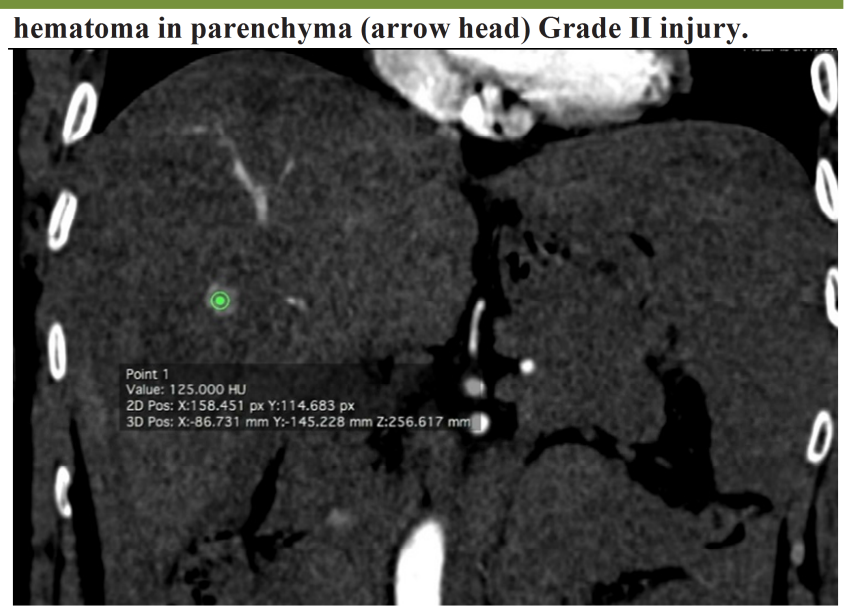

Figure 3: Coronal reformatted CT section showing active contrast extravasation in liver parenchyma (arrow head) in Arterial phase.

Active haemorrhage is identified in early arterial phase in contrast abdomen study as focal high-attenuation areas that represent a collection of extravasated contrast material secondary to arterial bleeding. ${ }^{[17]}$

In our study 39 cases showed positive CT findings of solid organ injury. Liver was the most commonly injured solid organ in 21 cases attributing to $54 \%$ of the injured cases followed by Splenic injury in 16 cases attributing to $41 \%$ of the injured and renal injuries in 11 cases attributing to $28 \%$ of the cases. Grading of injury was done according to AAST criteria. Out of the 39 cases, majority had Grade III and IV injuries (both $30 \%$ ), followed by grade II injury $(27 \%)$.Grade I injuries were seen in 3 cases even lesser than Grade V injuries (4).

Anderson W S et al, ${ }^{[13]}$ studied 68 patients out of which 47 patients underwentcomputed tomography for examination of abdominal injuries. Out of these 47 casesmajority of cases belonged to grade II constituting $45 \%$ of cases followed by grade IIIand grade IV with incidence of $21 \%$ \& $19 \%$ respectively. Grade I and grade V wasdiagnosed in 6 and 1 case respectively out of 47 cases with incidence of $13 \%$ and $9 \%$ each.

\begin{tabular}{|c|c|c|}
\hline \multicolumn{3}{|c|}{ Kidney injury scale } \\
\hline Grade $^{*}$ & $\begin{array}{l}\text { Type of } \\
\text { injury }\end{array}$ & Description of injury \\
\hline \multirow[t]{2}{*}{1} & Contusion & Microscopic or gross hematuria, urologic studies normal \\
\hline & Hematoma & Subcapsular, nonexpanding without parenchymal laceration \\
\hline \multirow[t]{4}{*}{ ॥ } & Hematoma & Nonexpanding perirenal hematma confirmed to renal \\
\hline & & retroperitoneum \\
\hline & Laceration & $<1.0 \mathrm{~cm}$ parenchymal depth of renal cortex without urinary \\
\hline & & extravagation \\
\hline \multirow[t]{2}{*}{ III } & Laceration & $\begin{array}{l}<1.0 \mathrm{~cm} \text { parenchymal depth of renal cortex without collecting system rupture or urinary } \\
\text { extravagation }\end{array}$ \\
\hline & Laceration & Parenchymal laceration extending through renal cortex, \\
\hline \multirow[t]{2}{*}{ IV } & & medulla, and collecting system \\
\hline & Vascular & Main renal artery or vein injury with contained hemorrhage \\
\hline \multirow[t]{2}{*}{ v } & Laceration & Completely shattered kidney \\
\hline & Vascular & Avulsion of renal hilum which devascularizes kidney \\
\hline \multicolumn{3}{|c|}{${ }^{*}$ Advance one grade for bilateral injuries up to grade III } \\
\hline
\end{tabular}




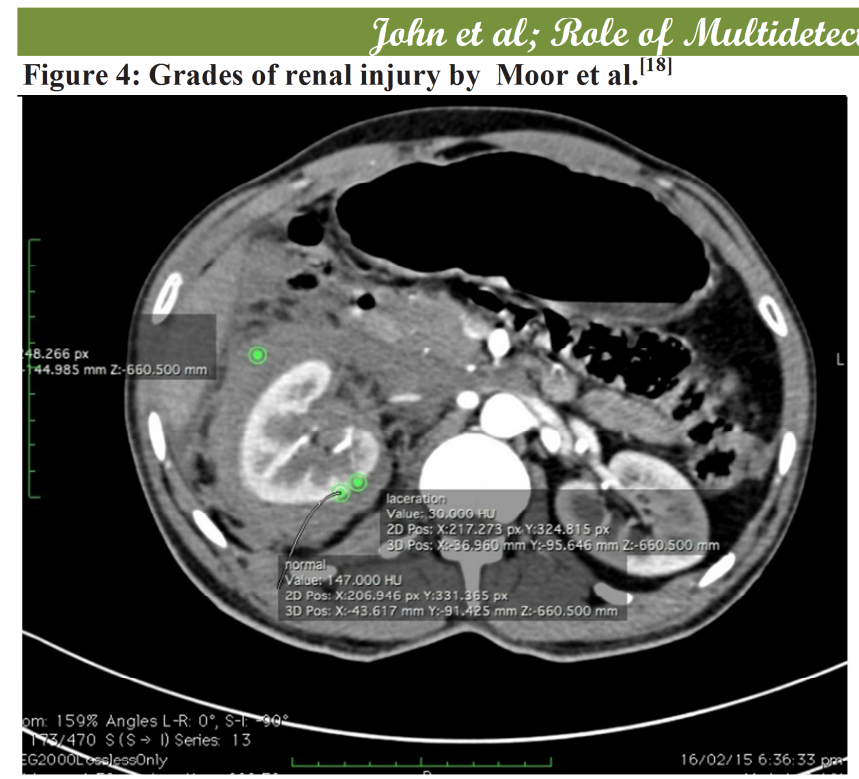

Figure 5: CECT axial section showing Renal injury. Laceration (black arrows), hematoma (white arrows)

Bowel and mesenteric injury were reported in 10 cases attributing to $25 \%$ of the total injured cases in our study. CT had a sensitivity, specificity, NPV, PPV of $75 \%, 96 \%, 0.90$ and $0.88 \%$ respectively.

A 2013 study found out that for detecting bowel and mesenteric injury the sensitivity and specificity was 55.33 and $92.06 \%$ respectively. The positive and negative predictive values were 0.61 and $.89 \%$ respectively. ${ }^{[14]}$

We had only two cases of adrenal injury both were diagnosed by $\mathrm{CT}$ accurately. Both cases presented as oval shaped hematomas on evaluation.

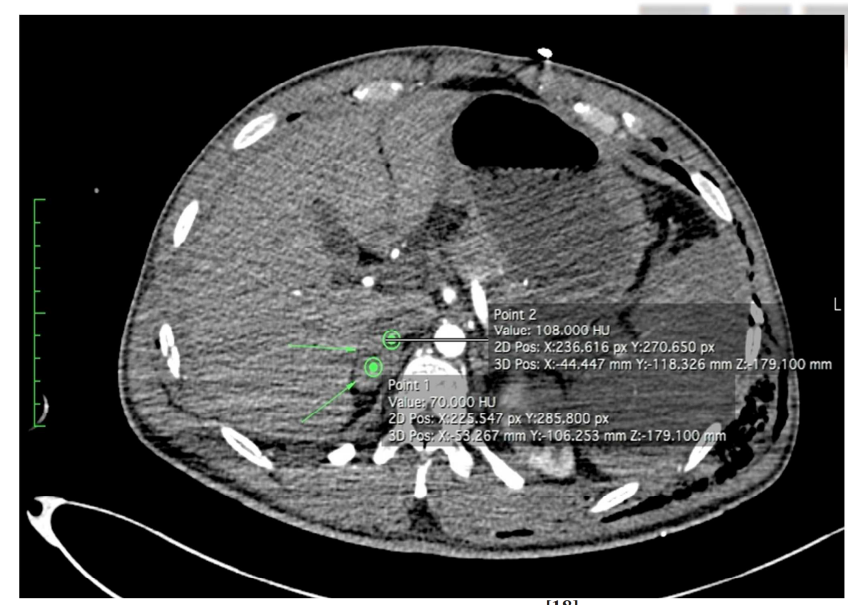

Figure 6: Adrenal hematoma (Grade V), ${ }^{[18]}$ injury

\begin{tabular}{|ll|}
\hline \multicolumn{2}{|l|}{ Adrenal organ injury scale } \\
\hline Grade $^{*}$ & Description of injury \\
\hline I & Contusion \\
\hline II & Laceration involving only cortex $(<2 \mathrm{~cm})$ \\
III & Laceration extending into medulla $(\geq 2 \mathrm{~cm})$ \\
\hline IV & $>50 \%$ parenchymal destruction \\
\hline V & $\begin{array}{l}\text { Total parenchymal destruction (including massive intraparenchymal hemorrhage) } \\
\text { Avulsion from blood supply }\end{array}$ \\
\hline${ }^{*}$ Advance one grade for bilateral lesions up to grade V \\
\hline
\end{tabular}

CF in Blunt Cladaminal Irouma

Figure 7: Adrenal gland injury scale by Moor et al. ${ }^{[18]}$

Table no: 3 Depicts the frequency of various grades of Injury in each organs as detected by MDCT

\begin{tabular}{|c|c|c|c|c|c|c|c|}
\hline \multicolumn{6}{|c|}{ Grade Of Injury } & \multirow{2}{*}{$\begin{array}{c}\text { Total } \\
\text { Number } \\
\text { Of Cases }\end{array}$} & \multirow{2}{*}{$\begin{array}{c}\% \\
\text { OF } \\
39\end{array}$} \\
\hline & I & II & III & IV & $\mathbf{V}$ & & \\
\hline Liver & 3 & 4 & 4 & 9 & 1 & 21 & $54 \%$ \\
\hline Spleen & 0 & 6 & 6 & 2 & 2 & 16 & $41 \%$ \\
\hline Kidneys & 0 & 3 & 4 & 3 & 1 & 11 & $28 \%$ \\
\hline Adrenals & 0 & 1 & 0 & 1 & 0 & 2 & $5 \%$ \\
\hline Pancreas & 0 & 0 & 1 & 0 & 0 & 1 & $2.5 \%$ \\
\hline
\end{tabular}

Burks DW et al, ${ }^{[15]}$ in their study on 1120 patients followed up for blunt trauma abdomen only $2 \%$ were finally diagnosed with adrenal injuries in CT. Nineteen (83\%) of theadrenal injuries appeared as discrete round to oval hematomas expanding the adrenal gland

Only one case of pancreatic fracture was reported in our study series. It was grade III on AAST criteria.

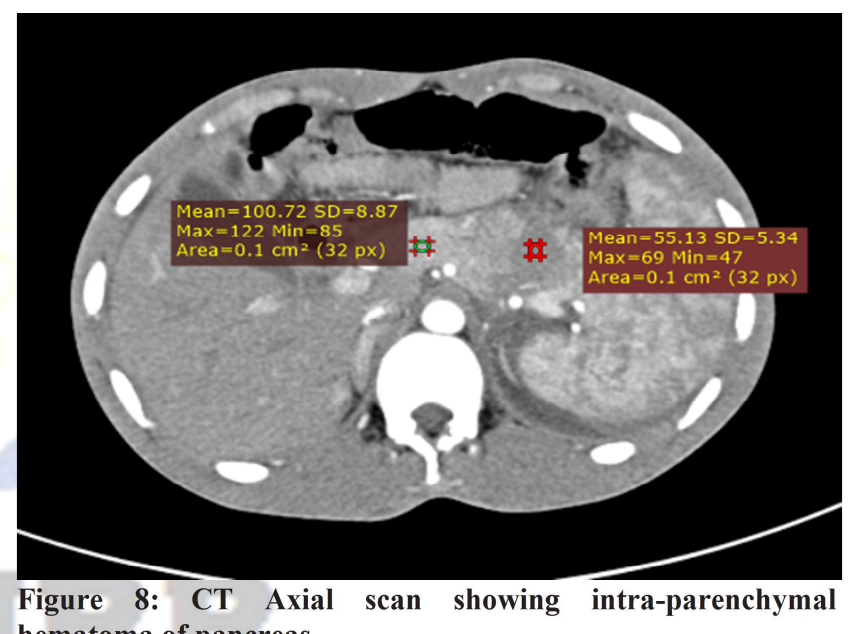

hematoma of pancreas.

Akhrass $\mathrm{R}$ et $\mathrm{al},{ }^{[16]}$ in their retrospective study on pancreatic trauma analysed 16188 cases of blunt trauma and found out that pancreas was involved in 1.1 per cent of patients with penetrating injuries compared to 0.2 per cent with blunt injuries.

The sensitivity, specificity, PPV and NPV for Bladder injury on comparison with CT findings were 100\%, 91.9\%, $50 \%$ and $100 \%$ respectively in our study.

\section{$\underline{\text { Summary }}$}

The detection rate sensitivity and specificity of solid organ injury by CT when compared with surgery were $97.5 \%$ and $100 \%$ respectively. Meanwhile the PPV and NPV were 100\% and $94.74 \%$ respectively. For Hollow Viscus Injury, on surgical correlation the sensitivity, specificity, NPV, PPV were $75 \%, 96 \%, 90 \%$ and $88.9 \%$ respectively.

Bladder injuries were analysed separately and we found out the sensitivity, specificity, NPV, PPV of 100\%, 91.9\%, 50\% and $100 \%$ respectively on comparison with surgical findings. All false positive cases were suspected to have extra-peritoneal type of bladder injury. On ascending urethrogram evaluation, 3 cases were found to have urethral 


\section{Tahn et al; Rale of Multidetector CI in Blunt Cladaminal J rouma}

injury.

Of the 56 cases admitted during the period of study, 49 cases were discharged on improvement of General condition and follow up done subsequently showed no further complications on any of these patients. 6 pateints died within 48 hours post admission, 2 cases died before intervention and all remaining were post-operative cases. 1 patient was discharged on request by the patient's relatives.One case was diagnosed to have splenic rupture with massive splenomegaly, which was evaluated further for cause of splenomegaly on follow up and was found to have Chronic Myeloid Leukaemia and he is now on Chemotherapy for the same.

\section{SENSITIVITY \& SPECIFICITY CHART}

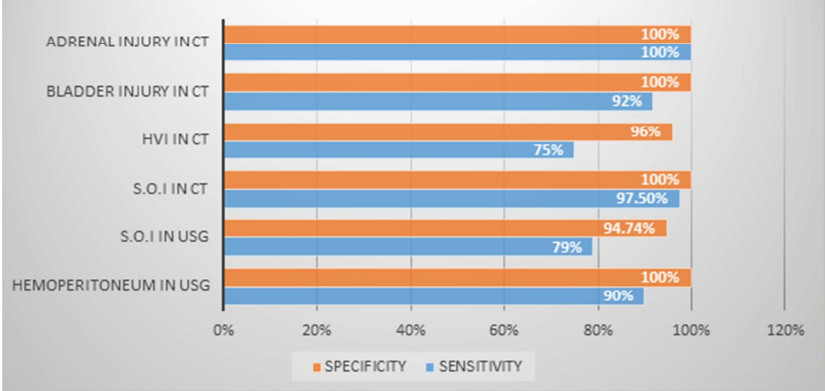

Graph 1: Bar diagram comparing sensitivity and specificity. S.O.I -SOLID ORGAN INJURY

\section{Conclusion}

MDCT is the golden standard for evaluation of blunt trauma abdomen. Prompt imaging in proper technique and expertise in reporting improves the detection rate and accuracy of all types of abdominal injuries associated with blunt trauma abdomen. While MDCT diagnosis of solid organ injury is highly accurate, the detection rate of Hollow viscus injury and bladder injury is not optimal. In examination where hemo-peritoneum in absence of solid organ injury is found high suspicion of hollow viscus injury should be made. Bladder injuries particularly extraperitoneal type should be further analyzed by ascending urethrogram in to confirm as well as to rule out urethral injury.

\section{References}

1. Root HD, Et al : Diagnostic peritoneal lavage: Surgery 1965;57:633
2. Livingston DH, Lavery RF et al. Admission or observation is not necessary after a negative abdominal computed tomographic scan in patients with suspected blunt abdominal trauma: JTrauma 1998;44:273-80.

3. Isenhour JL, Marx J. Advances in abdominal trauma. Emerg Med Clin North Am 2007;25:713-33

4. Hoff WS, Holevar M, et al; Fastern Association for the Surgery of Trauma. Practice management guidelines for the evaluation of blunt abdominal trauma: the EAST practice management guidelines work group. J Trauma 2002;53:602-15.

5. Salim A, Sangthong B, et al Whole body imaging in blunt multisystem trauma patients without obvious signs of injury: Arch Surg 2006;141:468-75.

6. Jorge A. Soto, et al Multidetector CT of Blunt Abdominal Trauma. RSNA Radiology; 65; 2012;673-693

7. Fang JF, Wong YC, et al Usefulness of Multidetector Computed Tomography for the Initial Assessment of Blunt Abdominal Trauma Patients : World J Surg 2006 Feb;30(2):176-82 42

8. Livingston DH, Lavery RF, et al. Admission or observation is not necessary after a negative abdominal computed tomographic scan in patients with suspected blunt abdominal trauma: results of a prospective, multi-institutional trial. J Trauma 1998;44:273-80.

9. Adult Routine Abdomen/Pelvis CT Protocols Version 1.1 August 7, 2015 by AAPM.

10. https://www.aapm.org/pubs/CTProtocols/documents/AdultAbdomen PelvisCT.pdf.

11. Lone GN, Peer GQ, Warn AK, Bhat AM, Warn NA. An experience with abdominal trauma in adults in Kashmir. JK Pract. 2001;8:22530 .

12. Nauman Al Qamari, Raza Sayani, Muhammed Ali, Muhammad Tamim Akhtar, Focused Sonography in Detecting Hemoperitoneum in Blunt Abdominal Trauma Patients, Correlation with Computed Tomography, International Journal of Medical Imaging. Vol. 1, No. 1, 2013, pp. 7-11.

13. Simon Fleming ,Ruth Bird et al Accuracy of Fast scan in blunt abdominal trauma in a major London Trauma centre; Internataional Journal of Surgery 2012;10:9,470-474

14. Stephan W. Anderson, Jose C. Varghese, Brian C. Lucey, Peter A. Burke, Erwin F. Hirsch, and Jorge A. Soto Blunt Splenic Trauma: Delayed-Phase CT for Differentiation of Active Hemorrhage from Contained Vascular Injury in Patients Radiology 2007 243:1, 88-95

5. Bhagvan, S., Turai, M., Holden, A., Ng, A., Civil, I. Predicting hollow viscus injury in blunt abdominal trauma with computed tomography. World J Surg. 2013;37:123-126

16. Burks DW1, Mirvis SE, Shanmuganathan K. Acute adrenal injury after blunt abdominal trauma :AJR Am J Roentgenol. 1992 Mar;158(3):503-7

17. Akhrass R, Yaffe MB, Brandt CP, Reigle M, Fallon WF Jr, Malangoni MA. Pancreatic trauma: a ten-year multi-institutional experience. Am Surg. 1997 Jul:63(7):598-604

18. Willmann JK, Roos JE, Platz A, et al. Multidetector CT: detection of active hemorrhage in patients with blunt abdominal trauma. AJR Am J Roentgenol 2002; 179:437-444.

19. Moore EE, Cogbill TH, Jurkovich MD, et al: Organ injury scaling: spleen and liver (1994 revision). J Trauma 38:323, 1995

Copyright: (C) the author(s), publisher. Asian Journal of Medical Radiological Research is an Official Publication of "Society for Health Care \& Research Development". It is an open-access article distributed under the terms of the Creative Commons Attribution Non-Commercial License, which permits unrestricted non-commercial use, distribution, and reproduction in any medium, provided the original work is properly cited.

How to cite this article: John PK, Khan SA, Ganesh K, Monteiro FNP. Role of Multidetector CT in Blunt Abdominal Trauma. Asian J. Med. Radiol. Res. 2018;6(1):30-34.

DOI: dx.doi.org/10.21276/ajmrr.2018.6.1.8

Source of Support: Nil, Conflict of Interest: None declared 